\title{
Anabases
}

ANABASES Traditions et réceptions de l'Antiquité

17 | 2013

Varia

\section{L'invention de la numismatique : des arts décoratifs aux sciences auxiliaires de l'histoire}

Jean Guillemain

\section{(2) OpenEdition}

1 Journals

Édition électronique

URL : https://journals.openedition.org/anabases/4116

DOI : 10.4000/anabases.4116

ISSN : 2256-9421

Éditeur

E.R.A.S.M.E.

Édition imprimée

Date de publication : 1 mars 2013

Pagination : 69-83

ISSN : 1774-4296

\section{Référence électronique}

Jean Guillemain, «L'invention de la numismatique : des arts décoratifs aux sciences auxiliaires de

l'histoire », Anabases [En ligne], 17 | 2013, mis en ligne le 01 avril 2016, consulté le 08 décembre 2022

URL : http://journals.openedition.org/anabases/4116 ; DOI : https://doi.org/10.4000/anabases.4116

Tous droits réservés 
Anabases 17 (2013), p. 69-83.

\section{L'invention de la numismatique : des arts décoratifs aux sciences auxiliaires de l'histoire}

JeAn Guillemain

On estimait à la Renaissance que les médaillons de pierre, de terre cuite ou de bois ornés de têtes de profil faisaient partie du décor de l'architecture antique. Parmi d'autres exemples, les élévations de bâtiments dans l'album de Giuliano da Sangallo ou l'édition de Vitruve par Cesariano (Côme, 1521) témoignent de cette idée. En réalité, il existe très peu de bas-reliefs romains présentant des têtes de profil, alors que les portraits de face sont assez courants, en particulier sur les sarcophages avec imago clipeata. En voulant illustrer les propos des auteurs anciens sur les portraits des grands hommes qui étaient exposés dans les places publiques et dans les bibliothèques, les lettrés des XVe et XVI ${ }^{\mathrm{e}}$ siècles ont pensé qu'il s'agissait aussi de bas-reliefs de profil (donc d'inspiration numismatique), sans doute parce qu'ils posaient l'équation : portrait $=$ médaille. Ainsi Étienne Dolet indique dans son dictionnaire de 1538 que imago est un synonyme du bas-latin megdala ${ }^{1}$, ce qui n'est évidemment pas la définition classique, mais explique le titre donné au plus célèbre recueil de portraits $\mathrm{du} \mathrm{XVI}^{\mathrm{e}}$ siècle, le Promptuaire des medalles (Lyon, 1553) qui est traduit en latin par Promptuarium iconum. Le mot "medalle " signifiait alors tout à la fois : pièce de monnaie antique ou contemporaine, médaille et grand médaillon avec effigie de face ou de profil, ces deux derniers objets étant des créations du Xve siècle. En se trompant, parce qu'ils projetaient dans l'Antiquité les formes qui faisaient partie de leur culture visuelle depuis quelques décennies, les hommes de la Renaissance n'en ont pas moins contribué à " restituer l'Antiquité ", dans la mesure où les artistes, puis les décorateurs et les illustrateurs ont

1 É. Dolet, Commentariorum linguae latinae tomus secundus, Lyon, 1538, col. 1516. 
appelé sur les monnaies l'attention des doctes, qui se sont appliqués à interpréter les revers monétaires. C'est dans ces circonstances qu'est née la numismatique, science auxiliaire de l'histoire ${ }^{2}$.

\section{Le médaillon avec tête de profil : l'invention d'un ornement "à l'antique »}

Au milieu du $\mathrm{XV}^{\mathrm{e}}$ siècle, les médaillons ornés de têtes de profil apparaissent en Italie dans les représentations peintes ou sculptées d'architectures imaginaires. Ces formes nouvelles sont conçues comme des agrandissements de médailles. Ainsi en 1443-1444 le peintre Giovanni Badile reproduit quatre médailles gravées par Pisanello et insère des citations de monnaies antiques (entre autres des empereurs Hadrien et Justinien) dans l'architecture qu'il invente pour le cycle de la Vie de saint Jérôme (Vérone, Santa Maria della Scala, fresques de la chapelle Guantieri) ${ }^{3}$. On connaît au Vatican les portes de bronze de Filarete, achevées en 1445. À Venise, vers 1450, un artiste florentin reproduit deux monnaies, de Faustine divinisée et de Galba semble-t-il, dans la frise qui surmonte la Mort de la Vierge dans la chapelle Mascoli à Saint-Marc. En Lombardie, Vincenzo Foppa peint en 1456 les Trois crucifiés vus à travers un arc de triomphe dont les écoinçons sont ornés de profils de césars affrontés (Bergame, Accademia Carrara). Avec le Milanais Bernardino Butinone, de telles représentations tendent à la reproduction réaliste de monnaies authentiques, comme le montrent l'emploi des couleurs bronze et or et les légendes monétaires rapportées plus ou moins fidèlement. On s'interrogera sur son choix de pièces citées dans la Vierge et l'Enfant avec les saints Jean Baptiste et Justine, vers 1495 (Isola Bella, Novare) : la médaille de François II de Carrare († 1406) voisine avec les monnaies de cinq empereurs des premiers siècles de notre ère, ce qui paraît confirmer la filiation entre médailles modernes et médaillons dans l'architecture feinte. Autre exemple, vers 1520, Giovanni Maria Falconetto reproduit assez fidèlement les portraits monétaires des vingt-quatre premiers empereurs romains, dans les écoinçons des arcades qui rythment la Salle $d u$ Zodiaque au Palais d'Arco (à Mantoue), mais l'art italien du XVI ${ }^{\mathrm{e}}$ siècle n'est pas aussi riche en médaillons que celui du Quattrocento tardif.

Après les peintres, les sculpteurs s’intéressent au motif du médaillon, dont ils font un ornement monumental. À la fin du Xve siècle, l'Italie septentrionale est le grand centre de la reproduction sculptée des monnaies romaines. En Lombardie, Giovanni Antonio Amadeo recouvre les monuments de médaillons à l'antique : après la Chapelle Colleoni à Bergame (1470-1475), il transforme la façade de la Chartreuse de Pavie en

2 Sur les premières générations de numismates, voir R. WeISs, The Renaissance discovery of classical Antiquity, Oxford, 1973, p. 167-179 ; F. HASKell, History and its images. Art and the interpretation of the past, New Haven et Londres, 1993.

3 R. Zerbato, "Giovanni Badile copia quattro medaglie del Pisanello : precisazioni ", Arte Veneta 21 (1977), p. 170-171. 
un gigantesque médaillier de plein air, où se succèdent les profils de tous les empereurs romains sculptés dans le marbre (1491-1498) ${ }^{4}$. L'architecture civile offre des portraits similaires, qu'on retrouve par exemple sur le portail du Palais Stanga (Crémone, aujourd'hui au Louvre). À Vérone, un artiste anonyme a sculpté des médaillons d'empereurs romains sur les pilastres de la Loggia del Consiglio, vers 1500 (fig. 1). À la même époque, à Gênes, de nombreux portails de palais sont ornés de profils affrontés, mais d'un travail parfois grossier qui ne permet pas d'identifier le prototype monétaire.

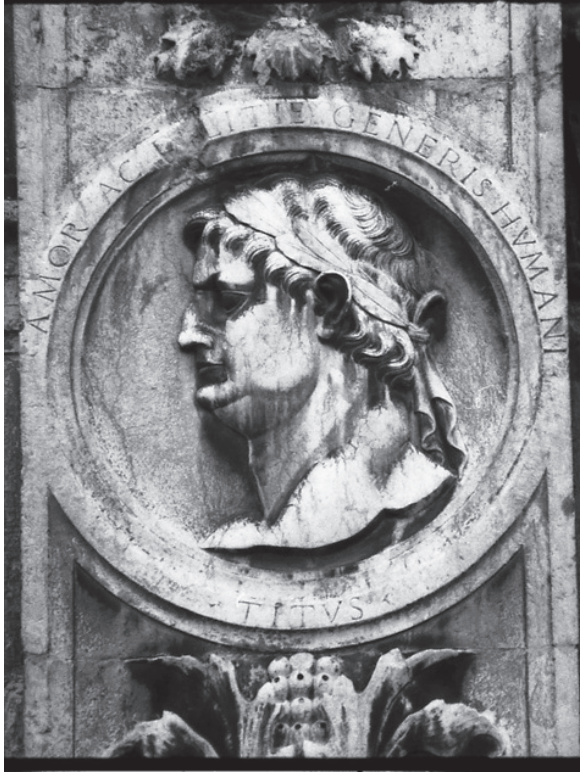

Fig. 1 : Médaillon de Titus, légendé Amor ac d[e]litie generis humani (Suétone, Tit., I), marbre, Vérone, Loggia del Consiglio, vers 1500 .

Le motif s'exporte dans les années 1500, en France et en Autriche notamment, en même temps qu'il perd en qualité plastique. Pour quelques médaillons de marbre importés d'Italie, comme à Gaillon, à Meillant ${ }^{5}$ ou Chenonceau, qui représentent fidèlement des empereurs romains d'après leurs monnaies, avec leur titulature, combien de bas-reliefs maladroitement sculptés dans la première moitié $\mathrm{du} \mathrm{XVI}$ e siècle, où s'affrontent le profil grossier d'un homme barbu et casqué et celui d'une femme sans expres$\operatorname{sion}^{6}$ ? On trouve des médaillons sur les supports les plus inattendus : pierres tombales, armures, vitraux, meubles, reliures (reliures " à froid " qui séduisent l'Europe du nord dans la première moitié $\mathrm{du} \mathrm{XVI}^{\mathrm{e}}$ siècle, mais aussi " reliures à la médaille " mode lancée par les Lyonnais sous Henri II), calvaires en bronze, etc. Ces médaillons sont presque toujours insérés dans le décor des encadrements, ce qui rappelle l'origine architecturale du motif. Dans les manifestations de ce qui fut une mode très largement répandue en Europe dans le deuxième quart $\mathrm{du} \mathrm{XVI}$ e siècle, le médaillon omniprésent conservait la fonction

4 A. Burnett, R. Schofield, "The medallions of the basamento of the Certosa di Pavia : sources and influence", Arte lombarda 120 (1997), p. 5-28.

5 B. De Chancel-Bardelot, "Hommes illustres en médaillons ", Berry Magazine 62 (2002), p. 20-24.

6 Rapide inventaire dans L. HAUTECEUn, Histoire de l'architecture classique en France [...], t. I, La formation de l'idéal classique, I, La première Renaissance (1495 à 1535-1540), Paris, 1963, p. 436-439. 
de ready made que lui avaient donnée les plasticiens du Quattrocento. C'est dans ce contexte que se développèrent les galeries de portraits ${ }^{7}$.

En 1517 parut à Rome, chez Jacopo Mazzocchi, le premier recueil imprimé d'effigies d'empereurs et d'hommes illustres fondé sur des sources numismatiques ${ }^{8}$. Le principal contributeur de cet ouvrage collectif était l'antiquaire Andrea Fulvio, dont on connaît par ailleurs les publications sur les ruines et la topographie de Rome. Dans ce livre de poche intitulé Illustrium imagines, où 204 notices biographiques accompagnées de portraits en médaillons sont présentées dans l'ordre chronologique, les " personnages illustres " sont pour la plupart les empereurs romains et romains germaniques, d'Auguste à Conrad le Salique, et les membres de leur famille. Janus, Alexandre le Grand et quelques célébrités de la République romaine les précèdent et quelques empereurs byzantins et princes carolingiens leur font suite. Les textes, qui tiennent en quelques lignes, se réduisent le plus souvent aux données généalogiques et passent sous silence les événements politiques et militaires, bien connus par Suétone, Dion Cassius, Hérodien et l' Histoire Auguste ou d'autres sources littéraires, auxquelles il n'est d'ailleurs jamais fait référence. En fait, la modernité de ce petit in-octavo réside dans les portraits, de profil, dont le sous-titre précise qu'ils sont tirés des monnaies antiques : Imperatorum et illustrium virorum ac mulierum vultus ex antiquis nomismatibus expressi, mais qui sont dans bien des cas des inventions de la Renaissance. Ainsi les visages des pères, mères et femmes des empereurs romains ont pour la plupart été dessinés pour l'occasion, parce qu'ils n'étaient pas connus par des monnaies antiques. D'autres portraits trouvent leur source dans des plaquettes de bronze. Par exemple l'effigie de Jules César, buste lauré à droite avec paludamentum retenu par une fibule inscrite SPQR, lituus dans le champ à gauche, étoile à droite et légende .DIVI.IVLI. (f. XVI des Illustrium imagines), représentée sur une plaquette du $\mathrm{XV}^{\mathrm{e}}$ siècle $^{9}$, a connu un grand succès : on la retrouve sur bien d'autres supports, tels que des gemmes, un manuscrit de la bibliothèque privée de Jules II daté de 1507, des reliures à la médaille (21 exemplaires recensés, de 1480

7 Sur l'édition numismatique à la Renaissance, voir J. GullLEmain, "L'antiquaire et le libraire : du bon usage de la médaille dans les publications lyonnaises de la Renaissance ", Travaux de l'Institut d'histoire de l'art de Lyon 16 (1993), p. 35-66 ; J.-L. FERRARY, Onofrio Panvinio et les antiquités romaines, Rome, 1996, p. 93-107 ; C.E. DeKESEL, Bibliotheca nummaria : bibliography of $16^{\text {th }}$ century numismatic books, Londres, 1997 ; J. CunNALLY, Images of the illustrious : the numismatic presence in the Renaissance, Princeton, 1999.

8 Illustrium imagines : imperatorum et illustrium nirorum ac mulierum uultus ex antiquis nomismatibus expressi et breues tituli cum inscriptionibus appositi per diuersos doctissimos uiros, sed pro maiori parte per Andream Fuluium diligentissimum antiquarium, quo emendatum correptumque est totum opus, Rome, 1517, disponible en ligne sur Gallica et sur Google books.

9 J. Pope-Hennessy, Renaissance bronzes from the Samuel H. Kress collection : reliefs, plaquettes, statuettes, utensils and mortars, Londres, 1965, $\mathrm{n}^{\circ} 270$. 
environ à 1558 environ) et des médaillons de marbre, l'un à Milan, Casa Botta, un autre, anépigraphe, au château d'Udine, dans le Salon du Parlement ${ }^{10}$.

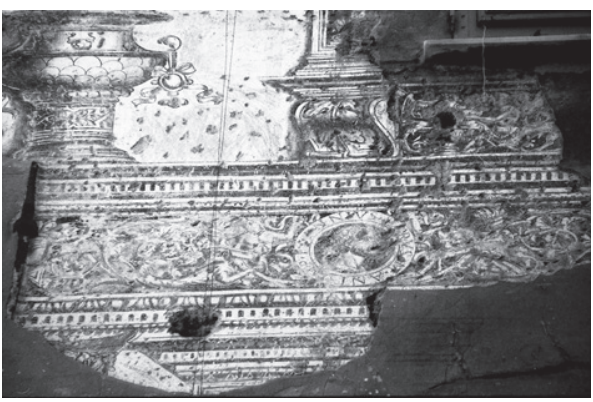

Fig. 2 : Médaillon d'Annius Verus, peinture murale, Gênes, Piazza Sauli 7, après 1517.

Les Illustrium imagines ne semblent pas avoir inspiré les peintres d'histoire, tandis que les hommes du livre et les décorateurs ont su en tirer parti. On entrevoit par exemple à Gênes, depuis la chute partielle du crépi d'un palais, piazza Sauli, une peinture murale qui comprend notamment une frise, entre les étages, où trois médaillons sont encore visibles, qui reproduisent des gravures du livre de 1517. Ces médaillons ne different de leur source que dans la mesure où le peintre a substitué les titres courants des pages aux légendes monétaires et les a inscrits en noir sur fond blanc. Ils se décri-

vent comme suit (dans l'état de 1997) : tout à droite de la façade, FAVST[IN]A[E] AVG.PII AVG.F., buste à gauche ; vers le milieu, [A]NNIVS [VE]RVS M.ANT [I] MP.P, buste à droite (fig. 2) ; à gauche du précédent, [AE]L[I]A [P]ETINA [CL.IMP.] VXO[R.], buste à gauche. Grâce au livre de Fulvio, il est possible de restituer le programme de la frise, qui devait se composer de deux séries de quatre médaillons, centrées sur les familles des « bons » empereurs Claude et Marc Aurèle. Ce sont, de gauche à droite :

- d'une part Drusus, père de Claude (buste à droite), Antonia, mère de Claude (buste à droite), Claude (buste à gauche), Aelia Petina, femme de Claude (buste à gauche) ;

- d'autre part Annius Verus, père de Marc Aurèle (buste à droite) (fig. 3), Domitia Calvilla, mère de Marc Aurèle (buste à droite), Marc Aurèle (mais la gravure reproduit une monnaie de Caracalla, buste à gauche), Faustine II, femme de Marc Aurèle (buste à gauche).

La fortune des gravures de ce livre a été considérable, comme l'indique le nombre des contrefaçons

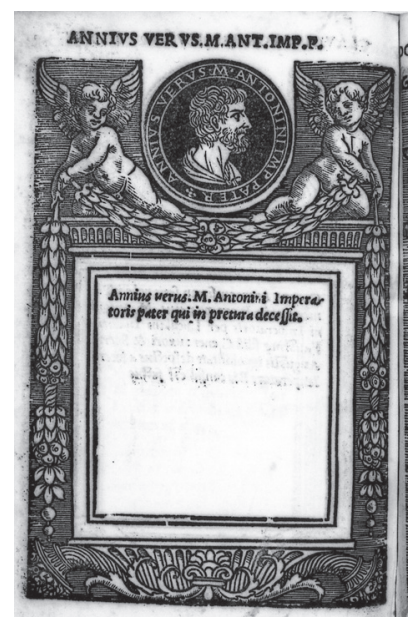

Fig. 3 : Médaillon d'Annius Verus, in Illustrium imagines, Rome, 1517, f. LXXV vº.

10 M. Vigerio, Decachordum christianum, 1507, exemplaire de dédicace, ms. Vat. Lat. 1125 ; A. HOBSON, Humanists and bookbinders : the origins and diffusion of the humanistic bookbinding, 1459-1559 with a census of historiated plaquette and medallion bindings of the Renaissance, Cambridge, 1989, p. 221-222, n 15-16 ; Burnett, Schofield, "The medallions ", p. 26 et fig. 69. 
et imitations auxquelles elles ont donné lieu dans le deuxième quart du XVI siècle, notamment en France et dans l'Empire, où les médaillons sculptés envahissaient les monuments, alors qu'ils passaient de mode en Italie. Le " libraire " François Juste a donné une copie conforme de ce livre (Lyon, 1524), dont l'illustration est de loin inférieure à celle de l'original, et Johann Huttich en a repris toutes les gravures mais avec des notices plus développées (Strasbourg, 1525, 1534 et contrefaçons à Lyon en 1550 et 1552). Dans l'épître au lecteur, Huttich précise qu'il a pour sa part composé un petit livre des vies des empereurs et que l'illustration numismatique lui a été imposée par son éditeur à l'intention des lecteurs qui ne disposeraient pas d'un médaillier.

Les éditeurs ont publié des livres de médailles jusqu'à la fin des années 1550, dans un format de plus en plus grand. Dans les Imperatorum romanorum verissimae imagines (Zurich, 1559, grand in-folio), André Gesner qui a pillé les publications lyonnaises de Strada et Rouillé suggère de faire afficher sur les murs des salles à manger ses planches de portraits des empereurs, renouant avec les origines architecturales de ce motif iconographique. Deux ans auparavant, Hubert Goltzius avait édité un livre au même format, dont les portraits d'une exceptionnelle qualité étaient accompagnés de quelques vers qui résumaient les faits marquants de la vie de chaque empereur. En fait c'est l'imprimeur lyonnais Balthazar Arnoullet, dans sa contrefaçon du recueil de Huttich en 1550, qui avait le premier associé effigie monétaire et tercet ou quatrain, à la manière des anciens Romains, mais aussi à l'imitation des livres d'emblèmes, dont la structure est communément : un motto (ou devise), une image, une épigramme. Pour ce qui est des références antiques, Cornelius Nepos rapporte que Pomponius Atticus avait composé un livre des hommes illustres, avec leurs effigies, sous lesquelles il avait inscrit quelques vers, et Pline évoque un travail semblable rédigé par M. Varron.

Les livres de médailles s'effacent dans les années 1560 devant les recueils de portraits in-folio publiés à Rome par le Bourguignon Antoine Lafréry et son petit-neveu Claude Duchet : série des jurisconsultes, par Marco Mantova Benavides en 1566, des papes, par Onofrio Panvinio en 1568, des hommes illustres par Achille Estaço en 1569 et par Fulvio Orsini en 1570. À partir de ce moment, les auteurs privilégient de plus en plus les reproductions de bustes et d'hermès, et cessent de donner aux portraits des hommes illustres la forme du médaillon, même lorsqu'ils sont effectivement tirés des monnaies et médailles.

La principale caractéristique des livres de médailles est que tous reproduisent en partie leur premier modèle. Les Illustrium imagines parues à Rome en 1517 ont ainsi fourni aux libraires de Venise, Lyon, Strasbourg et Zurich des dizaines de portraits des pères, mères et épouses des empereurs romains, qu'on retrouve inchangés jusque dans les dernières éditions du Promptuaire des medalles (1581). Vers le milieu du siècle tendent à s'imposer l'usage de la référence bibliographique et la justification par la physiognomonie d'un genre qui ne trouve pas son équilibre, entre recueil d'images et dictionnaire de biographie. Les portraits en médaillon n'illustraient que des ouvrages de vulgarisation - intitulés promptuaire, épitomé, libellus -, dont les nombreuses éditions indiquent qu'ils ont eu la faveur du public. Ces livres figurent dans bien des bibliothè- 
ques d'amateurs, comme celle de Claude de l'Aubespine, premier secrétaire d'État de Henri II, dont l'inventaire de 1571 révèle par ailleurs une grande proportion de livres d'histoire : on y trouve "Ung livre des anciennes medalles des empereurs, frippé ", "Trois Promptuaires de medalles, deux en françois et l'autre en latin » et l'édition de $1554 \mathrm{du}$ premier livre de Vico, "Omnium cesarum verissime imagines, en latin ». En revanche, les travaux des antiquaires ne semblent représentés que par "Les antiquités de Romme, de Andreas [Ful]vius ", dans ce cabinet de lecture qui ignore les recherches numismatiques de Costanzo Landi, Sebastiano Erizzo et Gabriele Symeoni, et celles pourtant bien diffusées de Guillaume du Choul ${ }^{11}$.

\section{Les revers monétaires : citations, emblèmes et iconographie}

$\mathrm{Au} \mathrm{XVI}{ }^{\mathrm{e}}$ siècle, les peintres italiens négligent les représentations de têtes de profil dans les médaillons, alors qu'ils utilisent de plus en plus les revers des monnaies romaines comme répertoire iconographique, pour le détail de leurs compositions. On connait, pour le milieu du XV siècle l'album de Jacopo Bellini et pour les années 1485-1488 les citations de revers monétaires par Domenico Ghirlandaio à Florence (Tombeaux des Sassetti à Santa Trinità, Massacre des Innocents à Sainte-Marie Nouvelle) ${ }^{12}$. À Rome, qui devient au début du siècle suivant le grand centre des études archéologiques, les collaborateurs de Raphaël copient des revers des monnaies romaines pour décorer de stucs les Loges du Vatican (1517-1519) ${ }^{13}$. Corrège s'en inspire de même pour les figures peintes de la Chambre de l'abbesse à Parme (vers 1519) ${ }^{14}$. Jules Romain, après avoir travaillé au Vatican avec Raphaël, ne procède pas autrement pour les stucs de la Sala del Sole e della Luna au Palais du Tè à Mantoue, où des dizaines de figures de revers sont enchâssées

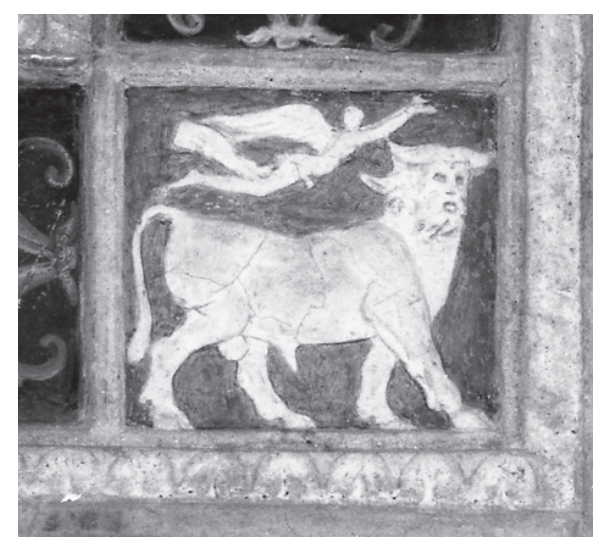

Fig. 4 : Jules Romain (atelier), Revers d'une monnaie de Neapolis (Naples, IV ${ }^{e}$-III siècle), stuc, Mantoue, Palais du Tè, Grotta, 1531-1534.

11 S. Le Clech-Charton, "Le cabinet de lecture de Claude de l'Aubespine à Bourges, ou la culture de la noblesse au XVI èècle ", Cahiers d'Archéologie et d'Histoire du Berry, 1996, Hors-série, Mélanges offerts à Jean-Yves Ribault, p. 221-225.

12 N. Dacos, "Ghirlandaio et l'antique ", Bulletin de l'Institut historique belge de Rome 34 (1962), p. 419-455.

13 N. Dacos, Le Logge di Raffaello : maestro e bottega di fronte all'antico, Rome, 1986.

14 E. Panofsky, La Camera di San Paolo du Corrège à Parme, trad. Marie-Claude Pouvesle, [Paris], 1996. 
dans des compartiments en forme de losange ${ }^{15}$ (fig. 4). À Vicence, Domenico Brusasorci isole lui aussi des figures de revers dans les compartiments de la voûte du Palais Chiericati (années 1550) ${ }^{16}$. De manière explicite, l'auteur de programmes Annibal Caro, passant en revue dans une lettre de 1562 les différentes monnaies antiques qui composent son médaillier, décrit la figure de la Justice nel qual modo la feci fare per la sepoltura di Paolo III ${ }^{17}$.

L'articulation entre les deux faces de la monnaie, le droit et le revers, s'établit lorsque le peintre accompagne le portrait d'une ou de plusieurs figures de revers, au second plan ou dans les encadrements. On rapprochera ici la série des Douze Césars peinte par le Titien à partir de 1537 pour le Palais ducal de Mantoue, celle du Véronais Felice Brusasorzi ${ }^{18}$, et celle de Jacopo Zucchi au palais Ruccellai à Rome : dans le premier cas les revers s'intègrent dans l'encadrement en stuc, dans les deux autres ils sont peints, et la légende est parfois reproduite. Vasari signale que le miniaturiste Giulio Clovio a peint un bon tableau nel quale è Traiano imperatore, secondo che si vede nelle medaglie, $e$ col rovescio della provincia di Giudea ${ }^{19}$. Cette tendance à associer à chaque empereur un seul revers, parmi des dizaines possibles, qui se cristallise dans la production de fausses monnaies antiques par les médailleurs padouans (notamment Giovanni da Cavino), confere aux revers monétaires une valeur emblématique. L'homme du Xvi siècle y reconnaît volontiers des figures énigmatiques accompagnées de textes lapidaires en langue savante, autrement dit les éléments constitutifs de l'emblème, qui est alors à la mode et dont le genre est codifié par Paolo Giovio. L'un des premiers numismates, Gabriele Symeoni, explique bien en 1561 que " ce que les anciens Romains mettoyent à l'envers des medailles, n'estoit que devises, et aucunefois sentences ${ }^{20}$ ".

Une variante du livre de médailles apparaît avec la publication d'Enea Vico, Le imagini con tutti $i$ riversi trovati et le vite degli imperatori tratte dalle medaglie et dalle historie degli antichi (Venise, 1548), qui illustre le monnayage des Douze Césars (c'està-dire de Jules César à Domitien). À la biographie et au portrait monétaire qu'on trouvait chez Fulvio et ses suiveurs, Vico ajoute pour chaque empereur un paragraphe sur sa physionomie, dont Suétone est la source, et quelques planches de revers moné-

15 F. VINTI, Giulio Romano pittore e l'Antico, Florence, 1995, p. 100-107.

16 G. BARIOLI, La moneta romana nel Rinascimento vicentino, Vicence, 1977.

17 A. Caro, Lettere familiari, éd. Aulo Greco, vol. III, Agosto 1559-Ottobre 1566, Florence, 1961, p. 126 (lettre no 671 à Fulvio Orsini).

18 L. Magagnato, "Felice Brusasorzi, Bernardino India, Sante Creara, Giambattista Rovedata ", in Cinquant'anni di pittura veronese (1580-1630), [Vicence], 1974, p. 62-64 et fig. 33-49.

19 G. Vasari, Le vite de' più eccellenti pittori, scultori e architettori, vol. III, Florence, Giunti, 1568, p. 853.

20 G. Symeoni, Description de la Limagne d'Auvergne en forme de dialogue, avec plusieurs medailles, statues, oracles, epitaphes, sentences et autres choses memorables et non moins plaisantes que proufitables aux amateurs de l'antiquité, Lyon, 1561, p. 3. 
taires, gravées sur cuivre. Ces derniers ne servent cependant pas à enrichir les notices biographiques. Le livre, qui est présenté comme une tentative de corpus numismatique, appartient donc encore au genre que Vico lui-même appellera par la suite libri di medaglie, dans lequel les monnaies antiques ne sont pas encore considérées comme des documents archéologiques, mais comme de simples illustrations.

C'est au milieu du $\mathrm{XVI}^{\mathrm{e}}$ siècle que se situent les débuts de la numismatique (bien que le mot soit plus récent), lorsque les antiquaires, qui avaient tous débuté en publiant des livres de portraits (Fulvio, Huttich, Du Choul, Vico, Strada, Goltzius), ayant retourné les monnaies, se donnent pour tâche d'interpréter les figures des revers. La décennie 1550 se signale par la publication soudaine à Lyon, à Venise et à Bruges d'études sur l'iconographie numismatique. Au dire de Goltzius (1557), c'était là une recherche méritoire : "Et jaçoit qu'une longue espace de temps on les ait tenues [les "renverses"] pour une chose de trop haut entendement, si est-ce touteffois qu'a present elles sont tenues en honneur des gens savans et lettrez. » En fait, c'est à Rome, ville où les peintres et les sculpteurs n'ont pas été intéressés par le motif du portrait en médaillon, que cette recherche a commencé. Dès 1540, Bernardino Maffei y avait entrepris un livre sur les revers monétaires. Il faut ici évoquer le programme de l'Académie Vitruvienne, de Rome, tel qu'il était défini par la fameuse lettre de Claudio Tolomei de 1542 : l'objectif était de préparer la publication d'une somme des connaissances sur l'Antiquité, en vingt livres, dont l'un était consacré aux monnaies. Ce projet collégial n'aboutit pas, mais suscita sans doute des travaux individuels, comme ceux du Lyonnais Guillaume du Choul, parent par alliance de Claudio Tolomei, dont les contemporains ont souligné l'excellence dans l'explication des revers monétaires ${ }^{21}$. Pour Antoine du Verdier, l'auteur de La bibliotheque (Lyon, 1585), Du Choul « a faict amas d'infinité de medailles et autres antiquailles et donné l'intelligence d'icelles, et de leurs revers, qu'il a par son estude et industrie diligemment tiree de plusieurs lieux et passages des historiographes anciens ${ }^{22}$ ".

21 Ginette Vagenheim voit aussi l'œuvre de l'antiquaire Pirro Ligorio comme une concrétisation du programme proposé par Claudio Tolomei. Voir sa contribution au présent dossier.

22 P.N. Pagliara, "Vitruvio da testo a canone ", in S. Settis (a cura di) Memoria dell'antico nell'arte italiana, vol. III, Dalla tradizione all'archeologia, Turin, 1986, p. 67-81 ; J. Guillemain, Recherches sur l'antiquaire lyonnais Guillaume du Choul (v. 1496-1560), thèse pour le dipl. d'archiviste paléographe, 2002, p. 104 et 243-255 ; ID., " L'exposition chez Guillaume du Choul ", in Le théâtre de la curiosité, Paris, 2008 (Cahiers V.L. Saulnier, 25), p. 167-182. 


\section{Étude de cas : l'illustration numismatique dans les pages de titre}

Dans les tout premiers livres imprimés, le texte commençait au recto du premier feuillet ; puis, pour protéger ce feuillet initial, on le fit précéder d'une feuille blanche. Les livres étaient alors vendus " en feuilles " et c'est le possesseur qui les faisait relier. Pour identifier les livres dans le commerce, le titre était inscrit sur cette page blanche, et il était parfois accompagné de gravures sur bois, du moins dans le cas d'ouvrages destinés aux publics les moins savants. Depuis les années 1485-1490, les informations essentielles sur le livre imprimé apparaissent sur la première page, qui change dès lors de fonction. La page de titre s'impose sous sa forme définitive dans le deuxième tiers du XVI siècle. Elle comporte les éléments suivants : nom de l'auteur, titre, marque de l'imprimeur ou de l'éditeur (qui reproduit l'enseigne de l'officine), nom de l'imprimeur ou de l'éditeur, lieu et date de publication. Un troisième nom apparait parfois sur la page de titre, celui du dédicataire, protecteur qui assume le rôle de garant auprès du lecteur ${ }^{23}$.

La page de titre joue donc un rôle publicitaire, analogue à celui de la couverture dans les ouvrages contemporains, visant à séduire l'acheteur. Et puisque les médaillons avec tête de profil sont à la mode, ils vont faire leur apparition sur les pages de titre, notamment lorsque celles-ci imitent les portails et les baies, où les formes numismatiques ne sont pas disposées au hasard, mais en quelques endroits bien normés (écoinçons de l'architecture de sensibilité gothique, pilastres des monuments "à l'antique ", allèges des fenêtres en France et des oriels dans le monde germanique) (fig. 5). À la fin $\mathrm{du} \mathrm{XV}^{\mathrm{e}}$ siècle, les enlumineurs italiens introduisent les portraits monétaires dans les encadrements architecturés dont ils ornent les pages initiales des manuscrits et des incunables. Quand ils ne sont pas peints de couleur bronze rehaussé d'or, à l'imitation des monnaies et de leur patine, ces profils sont des monochromes blancs sur fond rouge ou noir qui évoquent des gemmes. L'encadrement de type architectural simple, constitués de quatre bois assemblés, apparaît à la fin du Xve siècle. Un encadrement vénitien de 1493, présentant des pilastres ornés de médaillons avec des profils affrontés, a connu un grand succès. Il est repris à Paris par Josse Bade en 1511 (fig. 6), et immédiatement après copié par Nicolas de Barre, dont le tirage sert de modèle aux Lyonnais : Étienne Gueynard le fait graver en 1512, puis cède les bois à Claude Nourry en 1528, lequel le prête à plusieurs libraires; de sa veuve, il passe chez Pierre de Sainte-Lucie et finalement sert en 1540 pour une édition faite pour le compte des frères Frellon ${ }^{24}$. D'autres imprimeurs-libraires parisiens (Jean Petit, Galliot du Pré) et lyonnais utilisent des titres à

23 A. Charon-Parent, "Le livre de part et d'autre de Gutenberg. D'un monde à l'autre ", in F. Lestringant, M. Zink (dir.), Histoire de la France littéraire, t. I, Naissances, Renaissances: Moyen Âge-XVI siècle, Paris, 2006, p. 173-183.

24 H. BAUdRIER, J. BAUDRIER, Bibliographie lyonnaise : recherches sur les imprimeurs, libraires, relieurs et fondeurs de lettres de Lyon au XVI siècle, vol. XI, Lyon, 1914, p. 235-237. 
encadrement avec des têtes de profil en médaillon figurant sur les pilastres et colonnes, dans les écoinçons, les frises et les bases, puis la mode passe vers 1550. Le pic semble se situer dans les années 1520 et 1530, qui est aussi la période la plus féconde pour la sculpture de médaillons dans l'architecture française. En Espagne, le motif subsiste dans les années 1560.

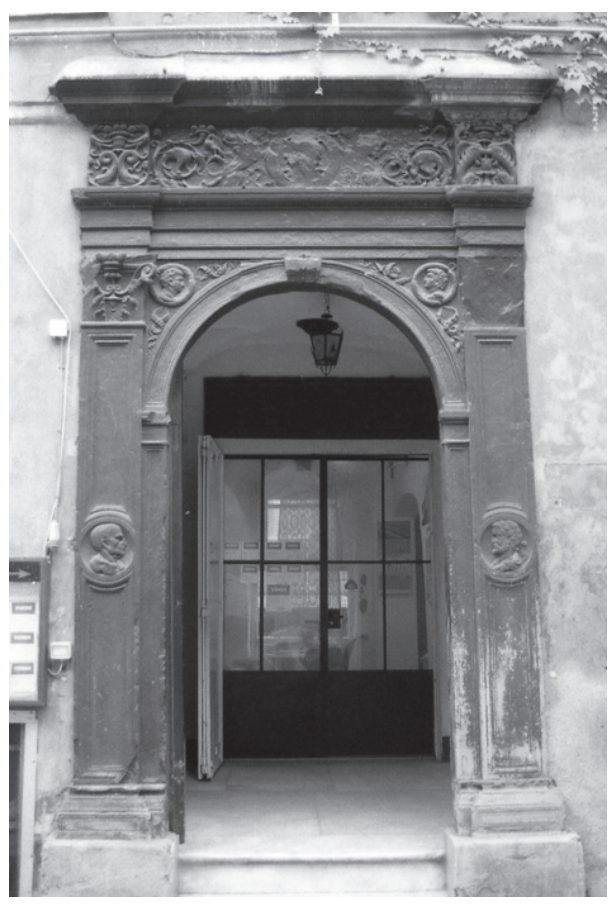

Fig. 5 : Portail de pierre, Gênes, Via S. Siro 2, $\mathrm{XVI}^{\mathrm{e}}$ siècle.

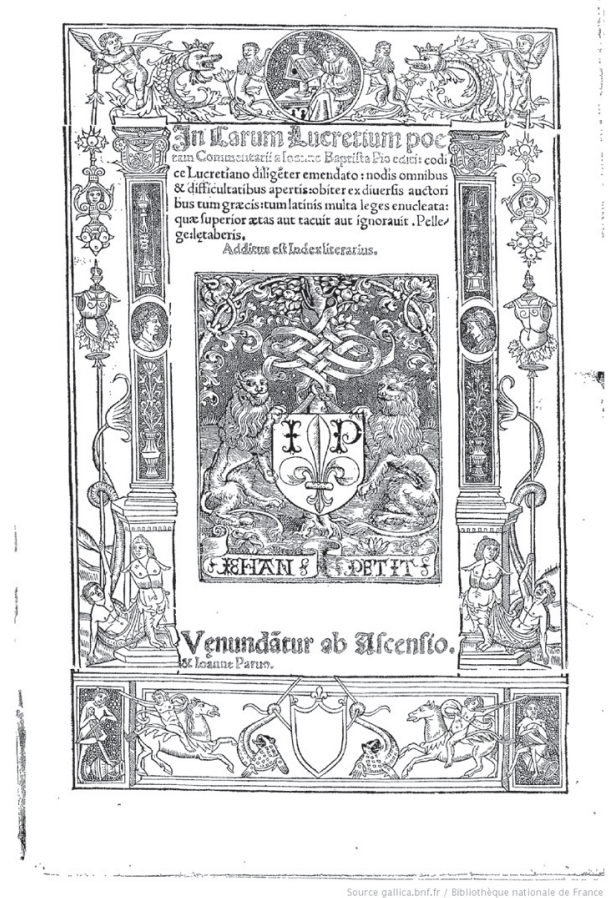

Fig. 6 : Encadrement employé par Josse Bade, In Carum Lucretium poetam commentarii a Joanne Baptista Pio editi, Paris, 1514.

Il convient de mentionner les portraits d'auteurs en médaillon sur les pages de titre lyonnaises - Dante (De Tournes, 1547), Érasme (De Tournes, 1558), Suétone (De Tournes, 1559), le portrait monétaire du roi pour la publication de ses ordonnances - avant de s'intéresser aux citations de revers monétaires dans les marques d'imprimeurs ou d'éditeurs.

La première marque typographique apparaît en 1457 sous la forme d'un bois gravé placé au colophon (c'est-à-dire à la fin) d'un livre imprimé par Fust et Schöffer. C'est l'équivalent d'une marque de fabrique ou de commerce, pour ne pas dire d'un logo. À la fin du Xv siècle, cette marque va se déplacer vers la page de titre, et l'embellir par ses figures. Du point de vue iconographique, beaucoup de marques faisaient 
allusion au nom du libraire selon le principe des armes parlantes (ou des calembours), ce qui les rendait immédiatement intelligibles (exemples de Michel Le Noir et l'homme noir, Galliot du Pré et la "gallée », Étienne Dolet et la doloire, la famille De la Porte avec Samson et les portes de Gaza, Sébastien Gryphe et le griffon, Jean Lecoq, etc.). D'autres marques, souvent accompagnées de devises, offraient un sens caché et s'apparentaient ainsi aux emblèmes, alors à la mode, lesquels n'étaient pas sans lien avec l'iconographie numismatique, comme nous l'avons vu.

La relation entre marques typographiques, emblèmes et revers monétaires antiques n’a guère été étudiée depuis les travaux de Guy de Tervarent (1958). La marque la plus célèbre, l'ancre aldine, trouve sa source dans le monnayage des Flaviens (des pièces d'argent et d'or de Titus et de Domitien au type du dauphin qui s'enroule autour d'une ancre). Alde Manuce devait l'interprétation de ce revers à Érasme, qui l'avait mise en relation avec la devise augustéenne Festina lente. Il l'utilisa à partir de 1495 et ses successeurs Paul et Alde II prolongèrent cette tradition pendant tout le XVI ${ }^{\mathrm{e}}$ siècle. La marque à l'ancre et au dauphin connut un grand succès et fut reprise par de nombreux imprimeurs-libraires en Italie, en France, en Hollande ${ }^{25}$. Les hommes du XVI e siècle en connaissaient parfaitement le sens. Après Paolo Giovio, Gabriele Symeoni, dans un passage signalé plus haut, explique en 1561 que

" ce que les anciens Romains mettoyent à l'envers des medailles, n'estoit que devises, et aucunefois sentences. Ne furent-ce pas deux sentences celles qui d'un mesmes sens, mais de figures diverses (comme le papillon et l'escrevisse, le dauphin et l'ancre) firent tailler Auguste et Tite empereur, voulans dire Festina lente? Au moyen de quoy je me resous [...] que toutes les armoiries et devises modernes soyent une mesme chose avec les medailles anciennes, se trouvant aussi bien aux unes comme aux autres diverses figures et $\operatorname{animaux}^{26}$ ».

Le premier exemple donné par Symeoni (le papillon et l'écrevisse) correspond lui aussi à une marque typographique, celle des Lyonnais Jean et François Frellon, qui l'utilisèrent de 1540 à 1568 avec le motto Matura (c'est-à-dire "Hâte-toi »), en l'inscrivant soit dans un cercle comme une monnaie (fig. 7), soit dans un cuir découpé. Puis Paul Frellon a repris cette marque entre 1593 et $1599^{27}$. Il est intéressant de relever que l'antiquaire lyonnais Guillaume du Choul possédait un exemplaire de la rare pièce d'or au nom d'Auguste qui représente au revers un crabe serrant un papillon dans ses pinces (fig. 8), nous le savons par Jacopo Strada qui documente cette pièce chez Du Choul en 1552-1553 et la décrit ainsi :

25 G. DE Tervarent, Attributs et symboles dans l'art profane : dictionnaire d'un langage perdu (1450-1600), Genève, 1997, p. 48.

26 Symeoni, Description, p. 3-4.

27 Baudrier, Bibliographie, vol. V, Lyon, 1901, p. 155 et 183 ; G. De Tervarent, Attributs, p. 168-169. 
Moderationis typus est. Cancer papilionem in aera subuolare nitentem anterioribus unguibus retinet. Inscriptio M. DVRMIVS III VIR. Hac figura illud significatum uoluit antiquitus, quod in prouerbis dicitur : Festina lente ${ }^{28}$.

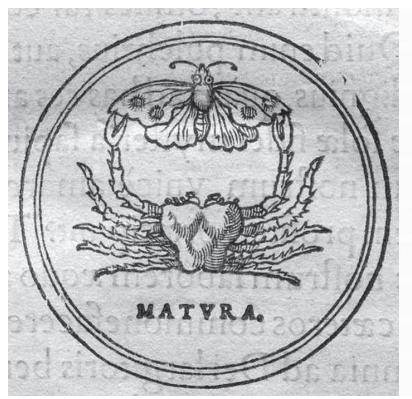

Fig. 7 : Marque de Jean II et François Frellon, Lyon, 1540-1551.
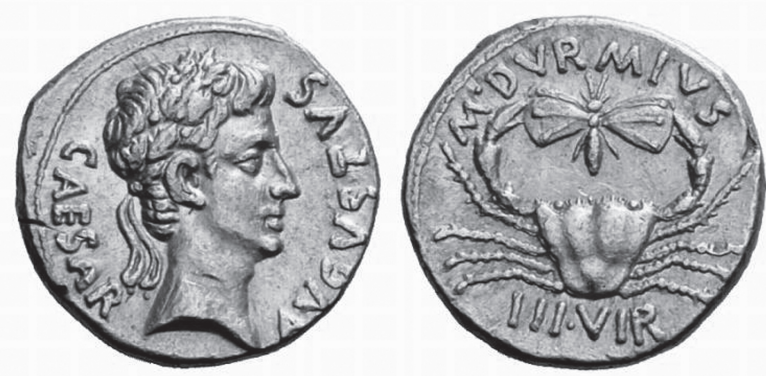

Fig. 8 : Aureus d'Auguste, Rome, 19 av. J.-C. (RIC 316), cliché Numismatica Ars Classica, vente 46 (2 avril 2008), $\mathrm{n}^{\circ} 475$.

Du Choul n'a pas illustré ce type dans son livre de 1556 sur la religion des anciens Romains, mais il a interprété une cornaline de sa collection, figurant Mercure assis sur un crabe, comme la "devise de la velocité qui est accompaignee de la tardivité ». Le thème faisait donc florès au milieu du XVI ${ }^{\mathrm{e}}$ siècle. Cette dernière image se retrouve en 1555 sur la marque du libraire Gabriel Cotier, qui publie un opuscule de Symeoni sur une pièce de la collection de $\mathrm{Du}$ Choul ${ }^{29}$. Un autre libraire lyonnais, Pierre Merant, a de même pris pour marque, en 1557, l'image d'une autre gemme de Du Choul, qualifiée cette fois de " devise de la providence ». Il est donc vraisemblable que Frellon, comme Merant et Cotier, ait trouvé le sujet de sa marque typographique (au crabe et papillon) dans la collection et les œuvres de Guillaume du Choul.

D'autres marques d'inspiration numismatique n'ont pas connu la même fortune que celle d'Alde Manuce. Je me suis proposé d'en identifier quelques-unes, en me fondant sur le corpus des publications italiennes du XVI ${ }^{\mathrm{e}}$ siècle présentées dans le site EDIT16 ${ }^{30}$.

28 J. Strada, $A$. A. A. numismatwn antiquorum $\triangle I A \Sigma K E Y H$, Vienne, Universitätsbibliothek, ms. III 160898, t. II, p. $173\left(\mathrm{n}^{\circ} 1\right)$.

29 G. du Choul, Discours de la religion des anciens Romains, Lyon, 1556, p. 155 ; BAUDRIER, Bibliographie, vol. IV, Lyon, 1899, p. 65.

30 Istituto Centrale per il Catalogo Unico delle biblioteche italiane e per le informazioni bibliografiche, EDIT16, Censimento nazionale delle edizioni italiane del XVI secolo, http:// edit16.iccu.sbn.it/web_iccu/ihome.htm (site consulté le 20 septembre 2012). 


\begin{tabular}{|c|c|c|c|c|}
\hline LIBRAIRE & LIEU & MARQUE & DATES & TYPE MONÉTAIRE $^{1}$ \\
\hline Alde et successeurs & Venise & Ancre et dauphin & $1502-1592$ & Titus RIC 112 \\
\hline Bascarini (Niccolò) & Venise & $\begin{array}{l}\text { Paon rouant (au } \\
\text { colophon) }\end{array}$ & $1543-1554$ & \\
\hline Blado (Antonio) & Rome & $\begin{array}{l}\text { Spes, avec motto Spes } \\
\text { augusta }\end{array}$ & $1543-1559$ & \\
\hline Boncompagno & Ferrare & Paon rouant & post 1585 & \\
\hline $\begin{array}{l}\text { Britannico } \\
\text { (Lodovico) }\end{array}$ & Brescia & $\begin{array}{l}\text { Rome et louve, dans } \\
\text { l'encadrement du titre }\end{array}$ & $1534-1557$ & $\begin{array}{l}\text { Vespasien } \\
\text { RIC } 442 \text { (sept } \\
\text { collines) }\end{array}$ \\
\hline $\begin{array}{l}\text { Al segno della } \\
\text { Concordia }\end{array}$ & Venise & Concordia & $1579-1599$ & $\begin{array}{l}\text { Ælius } R I C \\
\text { Hadrien } 428\end{array}$ \\
\hline $\begin{array}{l}\text { De Franceschi } \\
\text { (Francesco) }\end{array}$ & Venise & $\operatorname{Pax}$ & $1563-1596$ & \\
\hline Eliano (Vittorio) & Rome & Victoria & $1569-1570$ & Néron RIC 543 \\
\hline Giglio (Domenico) & Venise & Capricornes & $1553-1564$ & Auguste $R I C 126$ \\
\hline $\begin{array}{l}\text { Giordano } \\
\text { (Giacomo) }\end{array}$ & Padoue & $\begin{array}{l}\text { Aigle sur un globe, } \\
\text { entre deux serpents }\end{array}$ & $1562-1565$ & $\begin{array}{l}\text { Antoine } R P C \\
2201\end{array}$ \\
\hline Meda (Valerio) & Milan & Securitas perpetua & $1550-1558$ & $\begin{array}{l}\text { Dioclétien } R I C \\
83\end{array}$ \\
\hline $\begin{array}{l}\text { Nicolini da Sabbio } \\
\text { (Domenico) }\end{array}$ & Venise & Victoria & $1566-1593$ & Othon RIC 15 \\
\hline Pasquato (Lorenzo) & Padoue & Bonus Euentus & 1562 & Antonin RIC 676 \\
\hline Sanvito (Aniello) & $\begin{array}{l}\text { Venise } \\
\text { puis } \\
\text { Naples }\end{array}$ & Aigle sur globe & $1565-1575$ & $\begin{array}{l}\text { Vespasien } R I C \\
497\end{array}$ \\
\hline
\end{tabular}

1. $R I C=$ The Roman Imperial Coinage, Londres, 1923-...; RPC = A.M. BurnetT, M. Amandru, P.P. Ripollès, Roman provincial coinage, Londres, Paris, 1992-... Certaines figures de revers très courantes ne peuvent être rapportées à un monnayage en particulier : c'est le cas des divinités (Spes, Pax, etc.), mais aussi du paon rouant qui exprime l'apothéose des princesses.

Nous pouvons, pour conclure cet exposé, proposer une chronologie qui tienne compte de tous les types de citations numismatiques, quels que soient les supports et les aires géographiques. Sans prétendre fixer une périodisation rigide, nous retiendrons qu'il y eut deux ruptures importantes, qui se situent approximativement autour de 1510 et autour de 1560. La première phase (1440-1520), est celle qui voit l'éclosion du genre, avec les profils sculptés dans des médaillons qui ornent les monuments, ou les représentations peintes de monuments, en Italie septentrionale (et seulement septentrionale : les Romains ne participent que marginalement à ce mouvement). La deuxième phase (1510-1560) correspond 1) à l'abandon du motif par les Italiens et à sa récupé- 
ration par les Français, Autrichiens, Allemands, Suisses, Espagnols, éventuellement les Anglais, dans un mouvement de mode qui se traduit par la diversification des supports et surtout une forte baisse de qualité, 2) à la floraison des libri di medaglie, c'est-à-dire recueils de biographies illustrées de portraits monétaires, dont le fondement est celui de la physiognomonie, livres de portraits qui inspirent parfois décorateurs et sculpteurs, 3) à la vogue des encadrements avec médaillons sur les pages de titre, notamment en France. La troisième phase (1550-1600) pourrait être qualifiée de " débuts de la numismatique ", dans la mesure où les revers monétaires font l'objet d'études savantes, après toutefois que les peintres italiens y eurent trouvé un répertoire de formes. C'est alors que les imprimeurs-libraires, en France comme en Italie, saisissent tout l'intérêt des revers monétaires pour l'élaboration de leurs marques, témoignant ainsi de la place prise par les images numismatiques dans la culture visuelle de la Renaissance.

Jean Guillemain

Université Paris Descartes, Sorbonne Paris Cité

Conservateur de la bibliothèque H. Piéron

71, avenue Edouard Vaillant

F-92774 Boulogne-Billancourt

jean.guillemain@parisdescartes.fr 